\title{
EDITORIAL
}

\section{Frontiers of Physics: A new starting point}

I am most pleased with the growth of the journal Frontiers of Physics in China. Since its launch, the goal of the journal has been to become a truly high quality physics journal, and for such a goal, the editorial board and the editorial office have been committed to inviting papers on theoretical physics, experimental physics and applied physics. As of now, the journal has published many excellent papers from China, other areas of Asia, North America, and Europe, etc., and more and more authors are actively submitting their papers to the journal. Meanwhile, the journal has also organized several themed special issues, such as Iron-based Superconductors, Nanostructures and Nanodevices, Optical Crystals, Metamaterials and Plasmonics, etc., to summarize and report on the hot spots and exciting progress on various research fields in physics.

On 29 June 2010, we received good news that, after indexed by SCOPUS, INSPEC, Astrophysics Data System, etc., the journal was selected for coverage in Science Citation Index-Expanded (SCI-E), one of the most significant paper search engines used by scientists today. We believe that the indexing by SCI-E will further enhance the journal's development and the articles will be more readily searchable. The total cites of the journal articles have gradually increased, and more and more domestic and overseas physicists are being attracted. We would like to avail ourselves of this opportunity to thank the editorial board members for their hard work and selfless efforts moving the journal forward; we also would like to thank all the readers and contributors for their continuous support and thank all the peer-reviewers for their efforts keeping a high academic standard for the journal.

Since the first issue of 2011, the journal will be renamed as Frontiers of Physics, where "in China" will not appear. Undoubtedly, it will be a new start for the journal. The Journal has the admirably broad mission of serving not only as a forum in which Chinese researchers can present their results, but also as a touch point between Chinese physicists and those from outside China. We will continue to publish mainly invited review or mini-review articles focusing on the latest achievements of physics.

We will stick to the high editorial standards, the outstanding commitment to the authors, and the goal toward a genuinely top-tier physics journal. We cordially hope you can continue to support the journal and any suggestion and advice from you are welcome at any time.

On behalf of the journal and our team, as well as myself, I wish you all a happy and prosperous 2011.

Kind regards,

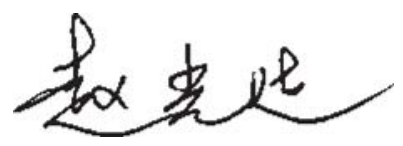

Kuang-ta CHAO

Editor-in-Chief of Frontiers of Physics

Professor of Physics, Peking University, China

E-mail: ktchao@pku.edu.cn 